\title{
Artefactos Explosivos Improvisados, Atención En Ambientes Austeros
}

\author{
${ }^{1}$ Erika TM Varona, ${ }^{2}$ Carlos AA Páez, ${ }^{3}$ Alejandro B Moreno
}

\section{RESUMEN}

Introducción: El trauma de guerra no convencional aún no está plenamente estudiado, se busca identificar tipos de lesiones en pacientes heridos por artefactos explosivos improvisados (AEI), en Colombia entre febrero de 2004 y mayo de 2014 que fueron atendidos por una de las unidades de atención quirúrgica móvil del ejército nacional de Colombia en ambiente austero (selva), con recursos limitados y remitidos posteriormente a hospital de cuarto nivel para manejo definitivo.

Materiales y métodos: Estudio observacional, descriptivo basado en una cohorte histórica. Se analizó la base de datos de pacientes civiles y soldados de las fuerzas militares de colombia que recibieron atención por los Grupos Avanzados de Trauma en ambientes austeros cercanos a las áreas de guerra, por artefacto explosivo. Se realizó revisión manual de las historias clínicas registrando las lesiones presentadas por pacientes al ingreso a la institución de salud de cuarto nivel. Se hace análisis con tablas dinámicas, frecuencias, tendencias, modas de los datos y se hace análisis de los mismos.

Criterios de inclusión: Pacientes que se encuentran registrados en base la de datos realizada por médicos participantes del grupo avanzado de trauma entre los años de 2004 al 2014 y que fueron víctimas por armas de fragmentación no convencionales.

Resultados: Se evaluó un total de 182 pacientes, Con promedio de edad de 23 años; Hubo 158 lesiones ortopédicas, la amputación fue la más frecuente en 105 pacientes (66.4\%), de las cuales $64.2 \%$ fueron infracondileas, $1.64 \%$ de miembros superiores y $0.54 \%$ supracondileas. $Y$ según rango militar; soldados $81.4 \%$ y Oficiales $6 \%$. Asociado al trauma oseo se presentó otras complicaciones como; Embolismo graso 1.64\%, Osteomielitis $2.94 \%$ Trauma acústico $3.85 \%$, Trauma ocular $3.29 \%$, Trauma facial $7.14 \%$, Trauma cráneo encefálico $0.54 \%$, Neuropatías $4.94 \%$, Sepsis por K. Pneumonia y E. Faecalis $2.19 \%$, con una mortalidad global de 1 paciente $(0,58 \%)$

\footnotetext{
${ }^{1}$ Interna Especial, ${ }^{2}$ Coronel, Cirujano General, Jefe and Profesor Ad Honorem, ${ }^{3}$ Residente de último año

${ }^{1}$ Área de Cirugía Cardiovascular y del Tórax, Hospital Militar Central, Universidad Militar Nueva Granada, Bogotá, Colombia

${ }^{2}$ Ejército Nacional de Colombia; Vascular y Angiólogo, Cirujano Cardiovascular; Área de Cirugía Cardiovascular y del Tórax Hospital Militar Central; Facultad de Medicina, Universidad Militar Nueva Granada. Bogotá, Colombia

${ }^{3}$ Cirugía General, Hospital Militar Central, Universidad Militar Nueva Granada, Bogotá, Colombia

Corresponding Author: Erika TM Varona, Interna Especial Área de Cirugía Cardiovascular y del Tórax, Hospital Militar Central, Universidad Militar Nueva Granada, Bogotá, Colombia Phone:+3022580309, e-mail: erikamendizabalv@gmail.com
}

Conclusión: En Colombia entre febrero de 2004 y Mayo del 2014 los AEI fueron la causa más frecuente de heridos de guerra generando lesiones osteomusculares con predominio de amputaciones infracondileas, con baja incidencia infecciones asociadas y mortalidad asociada.

Palabras clave: Artefactos explosivos improvisados, Austeros, Equipos quirúrgicos móviles, Heridos de guerra, Minas antipersona, Trauma.

How to cite this article: Varona ETM, Páez CAA, Moreno AB. Artefactos Explosivos Improvisados, Atención En Ambientes Austeros. Panam J Trauma Crit Care Emerg Surg 2017;6(2):61-67.

Source of support: Nil

Conflict of interest: None

\section{ABSTRACT}

Introduction: The trauma of unconventional warfare has not yet been fully explored. The aim of this article is to identify the types of lesions in patients injured by improvised explosive devices (IED) in Colombia between February 2004 and May 2014 and tended to by a mobile surgical team in an austere environment (jungle), with limited resources. The patients were later admitted to a level four hospital for definitive managemen.

Materials and methods: This is an observational, descriptive study based on a historical cohort. Database of civilian patients and soldiers of Colombian military forces injured by explosive device and who received care in Advanced Groups of Trauma in austere environments near areas of war was analyzed; manual review of medical records was performed by recording injuries presented by patients on admission to level four health institution. Analysis was done with pivot tables, frequencies, trends, means, and modes of the data and the data were analyzed thereof.

Inclusion criteria: Patients who were registered in the database by participating physicians of Advanced Group of Trauma between 2004 and 2014 and patients who were victims of unconventional weapons of fragmentation were included.

Results: A total of 182 patients with age range of 23 years were reviewed. There were 158 orthopedic complications; the most common amputation occurred in $66.4 \%$. Of these, infracondylar $64.2 \%$, supracondylar $0.54 \%$, and upper limbs $1.64 \%$. According to military rank, soldiers consisted of $81.4 \%$ and officials $6 \%$. The complications are as follows: Fat embolism 1.64\%, osteomyelitis $2.94 \%$, acoustic trauma $3.85 \%$, ocular trauma $3.29 \%$, facial trauma $7.14 \%$, traumatic brain injury $0.54 \%$, neuropathies $4.94 \%$, no trauma $4.39 \%$, sepsis by Klebsiella pneumoniae and Enterococcus faecalis $2.19 \%$, fatality $0.54 \%$.

Conclusion: In Colombia, between February 2004 and May 2014 IEDs were the most frequent cause of war wounded having musculoskeletal injuries, with predominance of infracondylar amputations with low infectious and deadly incidence.

Keywords: Austere, Improvised explosive devices, Landmines, Mobile surgical teams, Trauma, War wounded. 


\section{INTRODUCCIÓN}

En Colombia desde el año 1940 vive un conflicto armado en el cual frecuentemente se usa de manera irregular Artefactos Explosivos Improvisados (AEI) y Minas antipersona (MAP) $)^{1,2}$ que se definen como artefactos de explosión con químicos pirotécnicos o incendiarios que combinados con productos plásticos y/o metálicos aumentan su reacción química y generan un aumento en su onda expansiva (Figura 1). Debido a la manufactura no industrializada de la misma los grupos al margen de la ley han agregado elementos de tipo domestico buscando incrementar su potencial destructor como puntillas, tornillos e incluso excremento humano ${ }^{2}$, (Figura 2).

Esta intención de daño y el rápido avance por parte de las Fuerzas Militares en desarrollo de equipos de detección de AEI, generó un desarrollo por parte de los terroristas en los tipos de activación con el fin de hacerlos indetectables, como por ejemplo el uso de materiales plásticos y/o activación por contacto con émbolos de jeringas, contacto entre dos placas metálicas, ondas de radiofrecuencia, entre otros. ${ }^{2}$

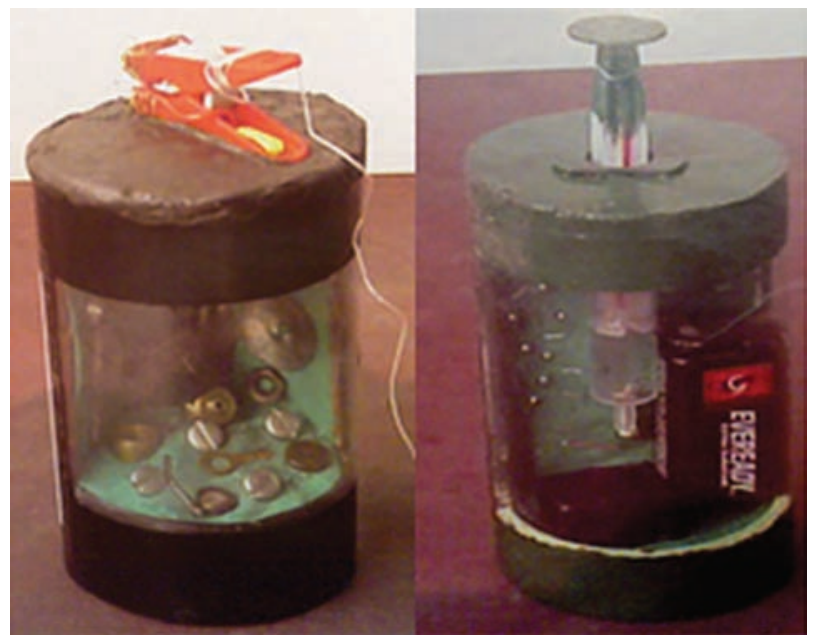

Figura 1: Artefactos explosivos improvisados

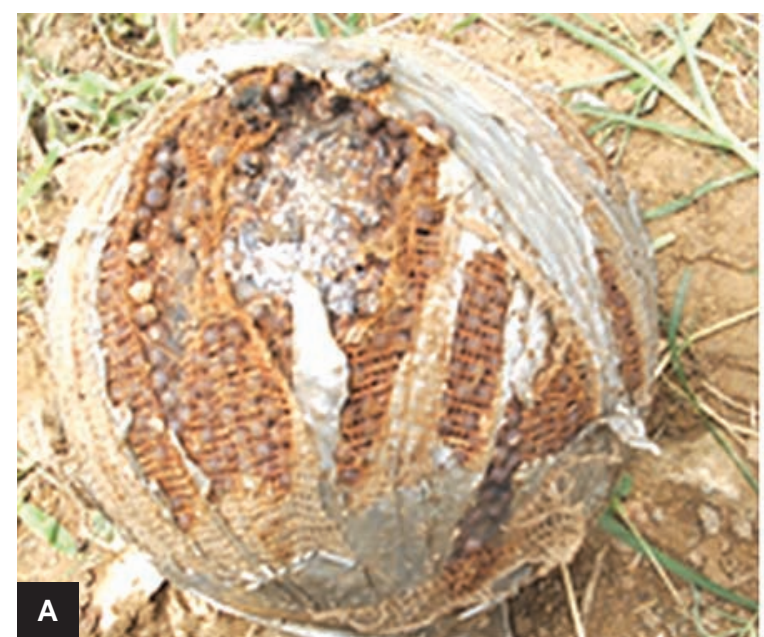

Diariamente en Colombia se difunden en informes militares y en prensa, los reportes hospitalarios de comunidades rurales en los que se reporta la muerte de combatientes y no combatientes a causa de estos artefactos bélicos, Actualmente se lleva una estadística de la frecuencia anual de eventos por este tipo de minas las cuales desde 1990 a septiembre de 2016 registra 34.512 casos de los cuales el 100\% son en áreas rurales; 6.707 accidentales y 27.895 incidentales. $^{3}$

Las Fuerzas Militares de Colombia crearon grupos especializados que recibieron el nombre de Grupos Avanzados de Trauma (GATRA), conformados por cirujanos generales, ortopedistas, anestesiólogos, instrumentadores, enfermeros, bacteriólogos y paramédicos, los cuales se desplazan a sitios cercanos de las área de enfrentamientos con terroristas, formando así equipos quirúrgicos que cuentan con material básico y personal esencial con el fin de brindar atención a los heridos cercana a las zonas de combate aumentando la supervivencia y disminuyendo las complicaciones por sangrado e infección; paralelo a esto se aumenta el nivel de riesgo de seguridad para el personal de salud y la exigencia de atención con recursos limitados a poli-traumatizados graves durante procedimientos de control de daños (Damage Control); (Figura 3 y 4); este tipo de grupos tienen una gran evidencia clínica desde la segunda guerra mundial conocidos como MASH (Mobile Army Surgical Hospital), CSH (hospitales quirúrgicos de combate $)^{4}$, en la mortalidad de heridos en combate ${ }^{4-6}$.

Durante la actividad de los grupos GATRA, se inició el control por medio de una base de datos desde 2004 hasta 2014 de pacientes, en la cual se registraron los datos de identificación, las diferentes escalas de trauma, el manejo inicial realizado en el área, razón por la cual se decide hacer un seguimiento de historias clínicas al ingresar a una institución médica de alto nivel (Hospital Militar Central, Bogotá, Colombia) y presentar un consolidado de las secuelas de una subunidad de estos grupos avanzados en trauma.

Figura 2A y B: Ejemplos de AEI, se evidencia contenido metálico bélico

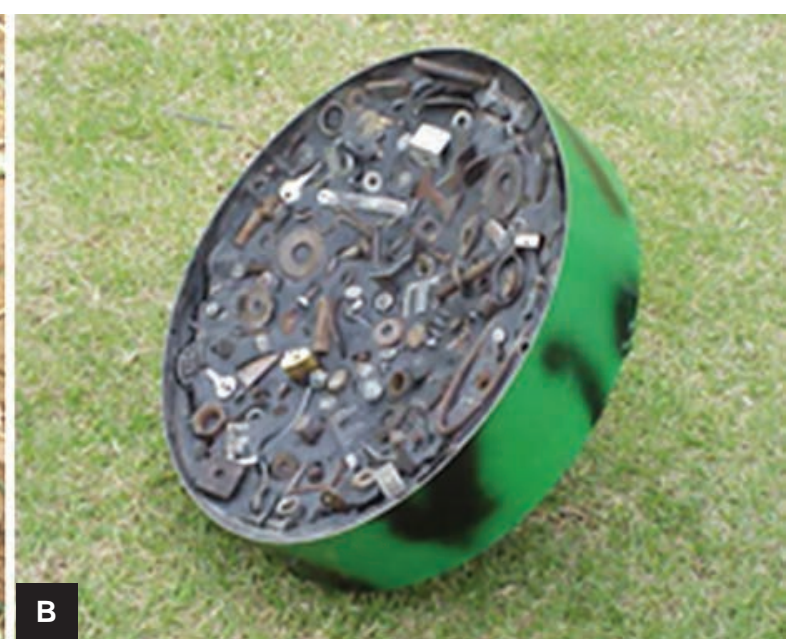



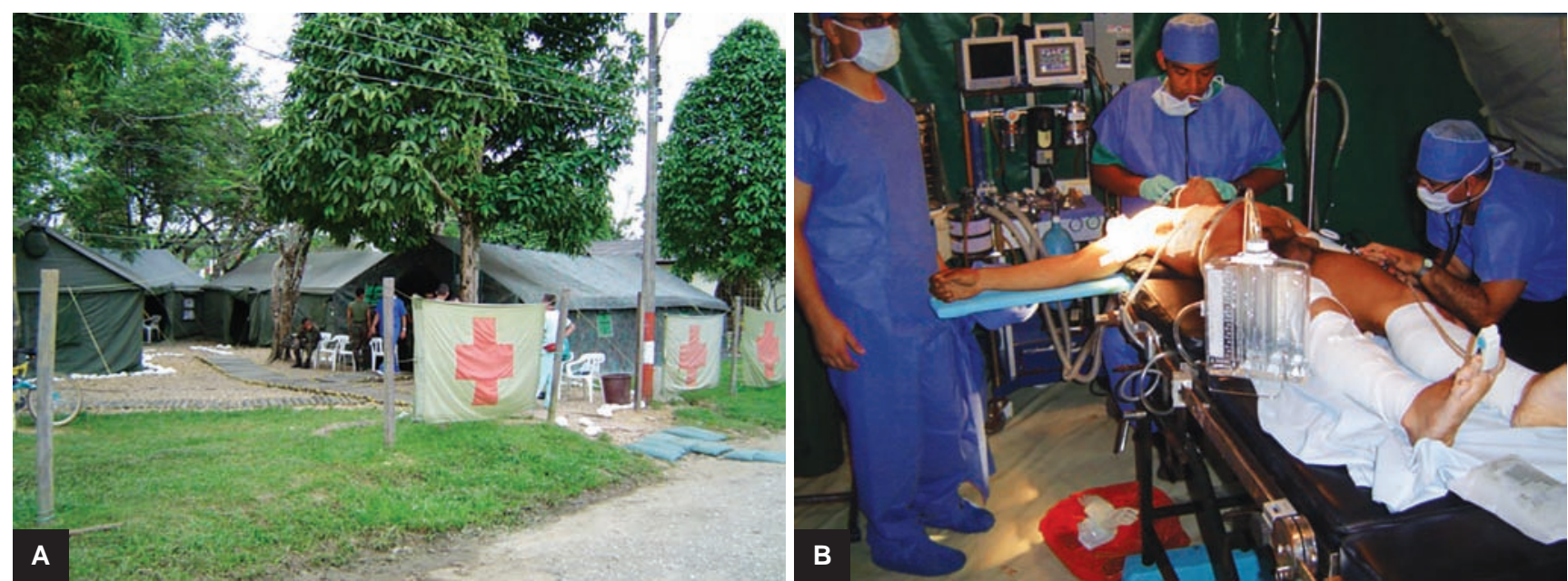

Figura 3A y B: Instalaciones GATRA
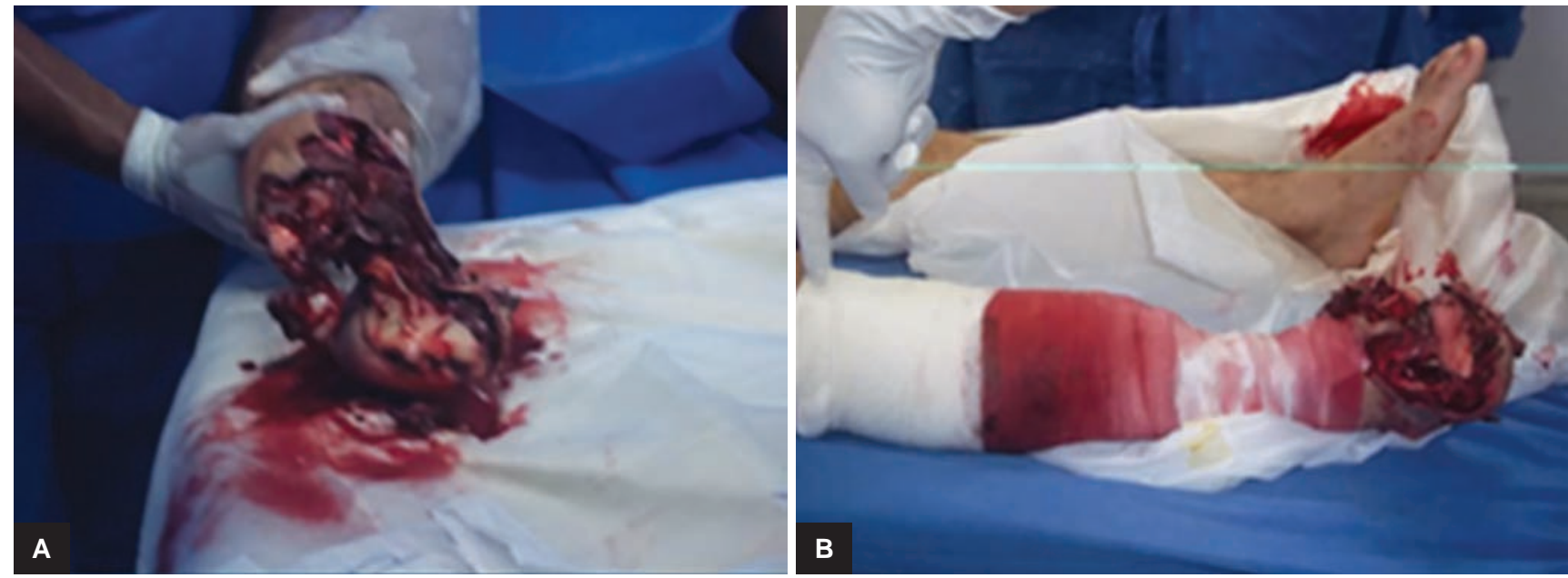

Figura 4A y B: Imágenes tomadas durante procedimiento quirúrgico de amputación tipo Syme, paciente atendido en Grupo GATRA entre 2004-2014: (A) Extremidad inferior derecha con amputación traumática por artefacto explosivo improvisado; (B) Extremidad inferior derecha en postoperatorio de control de daños.

\section{MATERIALES Y MÉTODOS}

Estudio observacional descriptivo basado en una cohorte histórica. Se analizó la base de datos de los heridos de guerra atendidos en una subunidad del GATRA, se utilizó filtro en tipo de arma "arma de fragmentación improvisada" la cual arrojo un resultado de 289 pacientes de los cuales se hizo una revisión manual con autorización previa del comité de historias clínicas y archivo del Hospital Militar Central y asegurando la confidencialidad de los datos personales de las historias clínicas utilizadas; logrando obtener la información completa en 182 pacientes, con las cuales se hace un compilado de las patologías presentadas y posibles complicaciones, para esto se utilizaron tablas dinámicas, frecuencias, tendencias, medias, promedios y medianas.

\section{Criterios de inclusión}

Pacientes registrados en la base de datos de una subunida del Grupo Avanzado De Trauma Entre los años de 2004 hasta el 2014. Pacientes que fueron víctimas de armas de fragmentación no convencional. Pacientes con historia clínica disponible en archivo de Hospital Militar Central, Bogotá, Colombia.

\section{Criterios de exclusión}

Pacientes de los cuales no se encontró historia clínica. Pacientes sin documento de identidad disponible.

\section{RESULTADOS}

Se analizó la base de datos obteniendo una población total de $n=288$, sin embargo al realizar la revisión de las historias clínicas se obtiene registro del $63.2 \%$, por lo cual en grupo final de estudio incluyo 182 pacientes, se registran las lesiones presentadas y las variables poblacionales se describen en la tabla 1.

Se evidencia un rango de edad entre los 20 a 41 años, para un promedio de edad de 25,9 años, moda 23 años, descrito en la Tabla 1. 
Tabla 1: Variables poblacionales

\begin{tabular}{lll}
\hline Población & $n=182$ & \\
\hline Masculino & 182 & $100 \%$ \\
Femenino & 0 & $0 \%$ \\
Militares & 181 & $99,40 \%$ \\
Civiles & 1 & $0,54 \%$ \\
Edad & & \\
$\begin{array}{l}\text { Rango de edad } \\
\text { Moda }\end{array}$ & 20 a 41 & Años \\
\hline
\end{tabular}

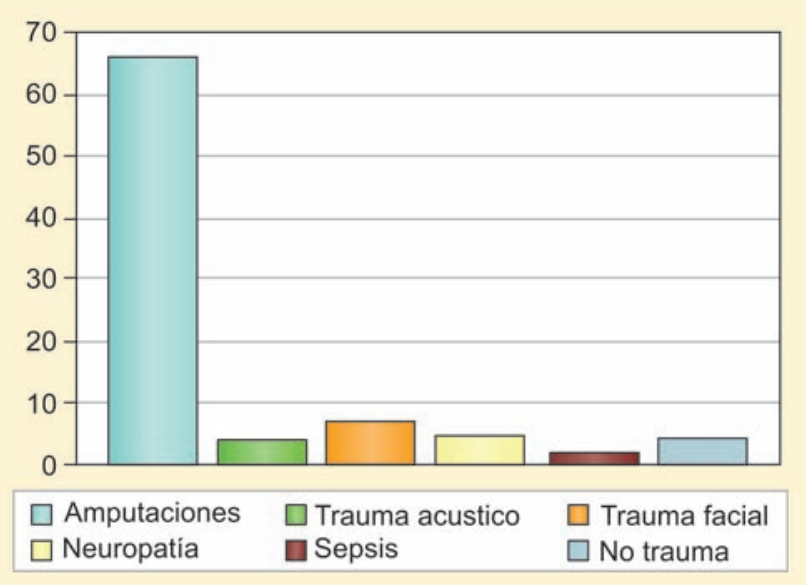

Gráfica 1: Tendencia de secuelas en pacientes afectados por AEI

Se identificaron158 lesiones ortopédicas, la amputación fue la más frecuente en 105 pacientes (66.4\%), de las cuales $64.2 \%$ fueron infracondileas, $1.64 \%$ de miembros superiores y $0.54 \%$ supracondileas (Gráfica 1 ).

Otras secuelas presentadas fueron embolismo graso $1.64 \%$, osteomielitis $2.94 \%$ Trauma acústico 3.85. Trauma ocular $3.29 \%$, Trauma facial $7.14 \%$ Trauma cráneo encefálico $0.54 \%$. Neuropatías $4.94 \%$, el $2.19 \%$ de los pacientes estudiados cursaron con sepsis por Klebsiella Pneumonia y Enterococcus Faecalis y solo hubo una muerte tardía $0.54 \%$, incluidas en la Gráfica 1.

\section{DISCUSIÓN}

En la actualidad Colombia se identifica como el único país en Latinoamérica que aún es víctima de este tipo de AEI y $\mathrm{MAP}^{4}$, creados por grupos al margen de la ley con fin de generar daño a las fuerzas militares y civiles, lo que ha llevado a catalogar según estándares internacionales una problemática grave en el país ${ }^{2}$, debido a la magnitud del trauma y evidenciando proyectos a nivel mundial de Hospitales quirúrgicos mobiles $^{4,5}$ en Colombia se crean los GATRA, lo que demuestra según este estudio y otros $^{6}$ un alto impacto en la reducción de las tasas de mortalidad e infección, sin embargo con alta incidencia de secuelas físicas por la frecuencia de amputaciones, con lo que se asume y por la edad promedio de 23 años en la población un alto impacto socioeconómico y cultural, que es directamente intervenido por la atención prioritaria en áreas austeras cercanas a los enfrentamientos entre fuerzas militares y grupos al margen de la ley.

Es así como se identifica la necesidad de equipos médicos entrenados en control de daños con mínimas dotaciones y que se desplacen hasta los puntos de mayor enfrentamiento con el fin de continuar la baja tasa de mortalidad que para este estudio fue tan solo del 0,54\% de la población. Cabe aclarar que pese a una larga data de guerra a nivel mundial y pese a los esfuerzos de mejorar las condiciones de atención en estas áreas rurales, las secuelas físicas se presentan en $95 \%$ de los pacientes.

Se considera de mucha utilidad continuar con registros y estudios estadísticos de secuelas en estas poblaciones con el fin de ejercer un plan de mejoramiento y entrenamiento en el personal médico que permita una atención oportuna e integral de las victimas reduciendo aún más las tasas de mortalidad, infección, amputaciones de varias extremidades en un mismo paciente o de regiones proximales de las mismas; lo que permitiría un impacto directo en la rehabilitación y reintegración de las víctimas.

\section{BIBLIOGRAFÍA}

1. Ministerio de postconflicto, derechos humanos y seguridad. Tipos de minas antipersona en Colombia. Available from: http://www.accioncontraminas.gov.co/accion/Documents/ Tipos\%20MAP,\%20MUSE\%20y\%20AEI.pdf

2. Ejército Nacional de Colombia, Historia de Artefactos explosivos improvisados en Colombia, 2015. Available from: https://www.ejercito.mil.co/?idcategoria $=384951$.

3. Vicepresidencia de la Republica. Plan nacional de acción contra minas. Estadísticas victimas mina antipersona 19902016. Available from: http://www.accioncontraminas.gov.co /estadisticas/Paginas/victimas-minas-antipersonal.aspx

4. King B, Jatoi I., The mobile Army surgical hospital (MASH): A military and surgical Legacy, J Natl Med Assoc 2005 May; 97(5):648-656.

5. Manring MM, Hawk, A, Calhoun JH, Andersen RC. Treatment of War Wounds: A Historical Review. Clin Orthop Relat Res 2009 Aug; 467(8):2168-2191.

6. Paez CAA, Arboleda FA, Mora FG, Gómez RB, Bernal LI, Gómez SP. Patrones de Trauma de Guerra en Colombia, Analisis del grupo quirúrgico avanzado de apoyo en trauma (GATRA) de las fuerzas militares de Colombia. Panam J Trauma Crit Care Emerg Surg 2012;1(2):61-67 


\section{COMENTARIO INVITADO}

\section{Artefactos Explosivos Improvisados, Atención En Ambientes Austeros}

Colombia lleva más de 50 años en un conflicto armado irregular y múltiples son las experiencias adquiridas en especial en la atención del trauma militar complejo.

El impacto en la atención médica de los heridos de una larga guerra irregular con la guerrilla y más recientemente con el narcotráfico y las pandillas emergentes, origino el desarrollo de una estrategia de atención médica que garantizara un tratamiento rápido, eficaz y optimo con la mayor probabilidad posible de supervivencia del herido y una rehabilitación satisfactoria.

La División médica de Las Fuerzas Militares de Colombia desarrollo el plan estratégico de atención médica denominado PANTERA basado en el análisis de tres factores a principios del año 2000; Una cifra preocupante de mortalidad inmediata en el campo de batalla del herido en combate del $32 \%$, a la ausencia de una atención médica especializada posterior al evento traumático y a el déficit de una logística de transporte médico con tiempos muy prolongados para la evacuación. Así como una mayor probabilidad de supervivencia del herido con una mortalidad del 6\% en el Hospital Militar Central como consecuencia de una atención medicamente especializada de alto nivel. ${ }^{1}$

El plan PANTERA se creó tomando como base el modelo de William Haddon quien en 1970 describió en New York una matriz de análisis para la atención en trauma en la que interrelaciona los factores promotores de la lesión; Humanos, Del medio ambiente y los Técnicos, en cada una de las tres posibles fases del evento; Pre, Durante y Post Trauma. Posterior a un año de planeación estratégica, el proyecto PANTERA se implementó en Febrero del 2004 con la participación de múltiples actores del área administrativa, militar, educativa y médica, y actualmente continúa estando activo.

En el periodo comprendido entre enero del 2005 a diciembre del 2010 se presentaron 8631 heridos de las fuerzas militares de Colombia en combate, hubo 2462 muertos en la escena de las operaciones militares (28.5\%). Las acciones de los equipos médicos y de evacuación permitieron una reducción muy significativa de la mortalidad en el campo de batalla; En el año 2005 hubo 531 muertos (35\%) y en el año 2010 hubo 425 (17\%). El entrenamiento del personal militar en los principios básicos de primeros auxilios y reanimación, con la aplicación de guías de manejo para el control de la hemorragia aguda y la profilaxis de la infección han sido un factor fundamental en la mejor probabilidad de la supervivencia del herido de combate. ${ }^{2}$

Desde enero del año 1999 hasta diciembre del 2010 el Hospital Militar Central de un total de más de 15000 heridos en combate atendidos, recibió 4233 pacientes críticamente lesionados (ISS >15), y la mortalidad hospitalaria disminuyo progresivamente del $5.2 \%$ al $2.3 \%$. Las tres primeras causas de mortalidad inmediata en el campo de batalla fueron las heridas en tórax (34\%), el desmembramiento por minas antipersonales (31\%) y las heridas en cráneo (17\%). ${ }^{2}$

En consecuencia el presente artículo realizado por la Dra. Mendizabal y colaboradores es importante al analizar un grupo de heridos de combate de una de las unidades GATRA y con el especial interés de revisar heridas secundarias a artefactos explosivos improvisados que ocasionan en su mayoría amputaciones, constituyéndose una de las series más grandes reportadas en la literatura mundial.

Efectuando el análisis de la técnica de guerra empleada por los grupos irregulares entre los años 2000-2005 el $80 \%$ de los heridos de las Fuerzas Militares de Colombia eran como consecuencia a heridas por arma de fuego de alto calibre y velocidad, en ese entonces la guerra se hacía en confrontación directa y con armamento convencional, pero el comportamiento de la técnica de guerra fue variando evitando el combate directo y para los años 2009-2010 el $75 \%$ de los heridos fue por acción de explosivos no convencionales y bombas antipersonales; Es por esa causa que la epidemiología de las heridas varió presentando en los últimos años pacientes con lesiones con una gran pérdida de tejido, con alto potencial de infección y con una alta tasa de amputaciones y desmembramientos. Esta variación de la táctica de guerra también ha sido experimentada por las fuerzas armadas de EEUU, principalmente en los conflictos de Afganistán e Iraq en donde también se utilizaron armas no convencionales y guerra de guerrillas. La disminución del número de soldados en combate por 100.000 durante los años 2005-2011 fue acompañada de un incremento en el número de combatientes amputados, cuadriplicándose la tasa de presentación. ${ }^{3,4}$ En el año 2010 el Sistema de Salud de Veteranos (VHA por sus siglas en ingles) de USA recibió cerca de 5000 nuevos pacientes amputados de los cuales el $20 \%$ correspondían a heridos en la guerra. ${ }^{5}$ A este respecto es útil recordar que uno de los grandes avances en la prevención de la muerte en el campo de batalla contemporáneo es el redescubrimiento del torniquete. 
Poco se ha escrito acerca de heridas por artefactos explosivo no convencionales y nuestro anhelo es que estos actos de barbarie no sean replicados en la humanidad. Sin embargo la guerra ha acompañado la misma existencia del hombre y nuestra realidad médica es que hay que estar preparados para ella.

\section{BIBLIOGRAFÍA}

1. Camargo J, Pérez LE, Franco C, Rodriguez E, Sanchez W. “Plan pantera” trauma militar en Colombia. Rev Colomb Cir. 2014:29; 293-304.

2. William Sanchez M.; Erwin Rodriguez-Garcia; David Rojas Tirado; Juan A Asensio. Results of the medical strategy for military trauma in Colombia. In: Asensio, Juan A.; Trunkey, Donald D., editors. Current therapy of trauma and surgical critical care. 2nd ed. Philadelphia: Elsevier; 2016. pp. 1-13.

3. Mason PE, Eadie JS, Holder AD. Prospective observational study of United States (US) Air Force Critical care Air Transport team operations in Iraq. J Emerg Med. 2011;41:8-13.

4. Eastridge BJ, Mabry RL, Seguin P, Cantrell J, Tops T, Uribe P, Mallett O, Zubko T, Oetjen-Gerdes L, Rasmussen TE, et al. Death on the battlefield (2001-2011): implications for the future of combat casualty care. J Trauma Acute Care Surg 2012 Dec;73(6 Suppl 5): S431-S437.

5. Krueger CA, Wenke JC, Ficke JR. Ten years at war: comprehensive analysis of amputation trends. J Trauma Acute care Surg. 2012 Dec;73(6 Suppl 5):S438-S444.

William Sanchez Maldonado Jefe Departamento de Cirugía

Hospital Militar Central, Universidad militar Nueva Granada Bogotá, Colombia 


\section{INVITED COMMENTARY}

\section{Artifacts, Improvised Explosive, Attention at Austeros Environments}

Colombia has been involved in an irregular armed conflict for more than 50 years, and there are many experiences gained in dealing with complex military trauma.

The impact on medical care of the wounded of a long irregular war with the guerrillas and, more recently, on drug trafficking and emerging gangs, led to the development of a health care strategy that would guarantee rapid, effective and optimal treatment with the greatest probability of wound survival and satisfactory rehabilitation.

The Medical Division of the Military Forces of Colombia developed the strategic health care plan called PANTERA based on the analysis of three factors at the beginning of the year 2000; A worrying figure of immediate mortality on the battlefield of the wounded in combat of $32 \%$, the absence of specialized medical attention after the traumatic event and the shortage of medical transport logistics with very long evacuation times. As well as a greater probability of survival of the wounded with a mortality of $6 \%$ in the Central Military Hospital as a consequence of medically specialized attention of high level. ${ }^{1}$

The PANTERA plan was created on the basis of the William Haddon model who in 1970 described in New York an analysis matrix for trauma care in which it interrelated the factors promoting the injury; Human, Environment and Technicians, in each of the three possible phases of the event; Pre, During and Post Trauma. After a year of strategic planning, the PANTERA project was implemented in February 2004 with the participation of multiple actors from the administrative, military, educational and medical areas, and is still active.

In the period from January 2005 to December 2010, 8631 people were injured by Colombian military forces in combat, with 2462 dead on the scene of military operations (28.5\%). The actions of medical and evacuation teams allowed a very significant reduction in mortality on the battlefield; In 2005 there were 531 deaths (35\%) and in 2010 there were 425 (17\%). The training of military personnel in the basic principles of first aid and resuscitation with the application of management guidelines for the control of acute hemorrhage and the prophylaxis of infection have been a fundamental factor in the best probability of the survival of the wounded in combat. ${ }^{2}$

Consequently, the present article by Dr. Mendizabal et al. is an important analysis of a group of combat wounded of one of the units GATRA and with special interest to review secondary wounds to improvised explosive devices that cause mostly amputations and constitutes one of the more significant series reported in the world literature.

Analyzing the warfare technique used by various groups between $2000-2005,80 \%$ of the wounded of the Colombian Armed Forces were as a consequence of gunshot wounds of high caliber and speed. At that time War was in direct confrontation and with conventional weapons. The behavior of the war technique avoided direct combat and for the years 2009-2010 75\% of the wounded was by unconventional explosives and anti-personnel bombs; It is for this reason that the epidemiology of wounds varied, presenting in the last years patients with lesions with a great loss of tissue, with high potential of infection and with a high rate of amputations and dismemberments. This variation of tactics of war has also been experienced by the US military, mainly in the conflicts in Afghanistan and Iraq where non-conventional weapons and guerrilla warfare were also used. The decrease in the number of soldiers in combat per 100,000 during the years 20052011 was accompanied by an increase in the number of combatants amputated, quadrupling the rate of presentation. ${ }^{3,4}$ In 2010, the US Veterans Health System (VHA) received about 5,000 new amputees, of whom 20\% were wounded in the war. ${ }^{5}$ In this regard it is useful to recall that one of the great advances in preventing death on the contemporary battlefield is the rediscovery of the tourniquet.

Little has been written about wounds by unconventional explosive devices and our longing is that these acts of barbarism are not replicated in humanity. However war has accompanied the very existence of man and our medical reality is that we must be prepared for it.

\section{REFERENCES}

1. Camargo J, Perez LE, Franco C, Rodriguez E, Sanchez WM : "Plan Pantera", Trauma militar en Colombia. Rev Colomb Cir. 2014:29;293-300.

2. Sanchez WM, Rodriguez-Garcia E, Tirado DR, Asensio JA : Results of the medical strategy for military trauma in Colombia. In Current Therapy of Trauma and Surgical Critical Care. Asensio \& Trunkey (ed.s) Elsevier 2016: Pages. 7-13.

3. Mason PE, Eadie JS, Holder AD. Prospective observational study of United States (US) Air Force Critical care Air Transport team operations in Iraq. J Emerg Med. 2011;41:8-13

4. Eastridge BJ, Mabry RL, Seguin P, et al. Death on the battlefield (2001-2011): implications for the future of combat casualty care. J Trauma Acute Care Surg 2012;73:Suppl:S431-S437.

5. Krueger CA, Wenke JC, Ficke JR. Ten years at war: comprehensive analysis of amputation trends. J Trauma Acute care Surg. Surg. 2012 Dec;73(6 Suppl 5):S438-S444. 